Pacific

Journal of

Mathematics

ISOMORPHISM THEOREM ON LOW DIMENSIONAL LIE ALGEBRAS

Jang-Ho Chun, Takeshi KaJiwara, And Jong-Sook LeE

Volume 214 No. 1

March 2004 


\title{
ISOMORPHISM THEOREM ON LOW DIMENSIONAL LIE ALGEBRAS
}

\author{
Jang-Ho Chun, TAKeshi Kajiwara, And Jong-Sook LeE
}

Let $\mathfrak{g}$ (resp. $\left.\mathfrak{g}^{\prime}\right)$ be a Lie algebra of dimension $d \leq 3$ (resp. of finite dimension) over a field $k$ of characteristic $\neq 2$. We prove that $\mathfrak{g}$ is isomorphic to $\mathfrak{g}^{\prime}$ as Lie algebras over $k$ if and only if the enveloping algebra $U(\mathfrak{g})$ of $\mathfrak{g}$ is isomorphic to $U\left(\mathfrak{g}^{\prime}\right)$ as $k$-algebras.

\section{Introduction.}

In this article, we study the isomorphism theorem on Lie algebras of dimension $\leq 3$. Our goal is the following theorem:

Theorem 1.1. Let $\mathfrak{g}\left(\right.$ resp. $\left.\mathfrak{g}^{\prime}\right)$ be a Lie algebra of dimension $d \leq 3$ (resp. of finite dimension) over a field $k$ (of characteristic not equal to 2 ). Then $\mathfrak{g}$ is isomorphic to $\mathfrak{g}^{\prime}$ if and only if the universal enveloping algebra $U(\mathfrak{g})$ of $\mathfrak{g}$ is isomorphic to the one $U\left(\mathfrak{g}^{\prime}\right)$ of $\mathfrak{g}^{\prime}$.

For a Lie algebra of dimension 1 or 2 , the theorem is clear by classification of low dimensional Lie algebras [3, I.4]. Malcolmson [4] proved the isomorphism theorem for 3-dimensional simple Lie algebras by using their Killing forms. We describe the simplicity of a 3-dimensional Lie algebra in terms of its enveloping algebra. To complete the isomorphism theorem on 3dimensional Lie algebras, we prove the theorem for non-simple Lie algebras of dimension 3 .

Notation. We denote by $\sigma=\sigma_{\mathfrak{g}}: \mathfrak{g} \rightarrow U(\mathfrak{g})$ a canonical map from a Lie algebra to its enveloping algebra $U(\mathfrak{g})$.

\section{Preliminaries on enveloping algebras.}

We prove some preliminary properties on the enveloping algebra $U(\mathfrak{g})$.

Proposition 2.1. The two-sided ideal $I_{\mathrm{com}}$ generated by $\{[a, b]:=a b-b a \in$ $U(\mathfrak{g}) ; a, b \in U(\mathfrak{g})\}$ is equal to the one $I_{[\mathfrak{g}, \mathfrak{g}]}$ generated by $\sigma([\mathfrak{g}, \mathfrak{g}])$.

Proof. We have only to verify $I_{\text {com }} \subset I_{[\mathfrak{g}, \mathfrak{g}]}$. Since $\sigma\left(g_{1}\right) \sigma\left(g_{2}\right) \cdots \sigma\left(g_{s}\right)\left(g_{i} \in\right.$ $\mathfrak{g})$ generate $U(\mathfrak{g})$ as a $k$-vector space, it is enough to show that

$$
\left[\sigma\left(g_{1}\right) \cdots \sigma\left(g_{s}\right), \sigma\left(h_{1}\right) \cdots \sigma\left(h_{r}\right)\right] \in I_{[\mathfrak{g}, \mathfrak{g}]}
$$


for $g_{i}, h_{j} \in \mathfrak{g}$. It follows from the formula

$$
\left[g, h h^{\prime}\right]=[g, h] h^{\prime}+h\left[g, h^{\prime}\right] \text { for } g, h, h^{\prime} \in U(\mathfrak{g}) .
$$

Proposition 2.2. In the notation of Proposition 2.1, we have a canonical isomorphism $U(\mathfrak{g}) / I_{\text {com }}=U(\mathfrak{g}) / I_{[\mathfrak{g}, \mathfrak{g}]} \rightarrow U(\mathfrak{g} /[\mathfrak{g}, \mathfrak{g}])$ as k-algebras.

Proof. See $[\mathbf{2}, 2.2 .14$, p. 72$]$. By the functoriality of $U(\mathfrak{g})$ with respect to $\mathfrak{g}$, we have a canonical $k$-algebra homomorphism $\varphi: U(\mathfrak{g}) \rightarrow U(\mathfrak{g} /[\mathfrak{g}, \mathfrak{g}])$. Since $\sigma(\mathfrak{g})$ generates $U(\mathfrak{g})$ as $k$-algebra, the homomorphism $\varphi$ is surjective. On the other hand, every (Lie algebra) homomorphism from $\mathfrak{g}$ to the Lie algebra associated to a commutative ring factors through $\mathfrak{g} /[\mathfrak{g}, \mathfrak{g}]$. Since $U(\mathfrak{g}) / I_{\text {com }}$ is commutative, we have a $k$-algebra homomorphism $\psi: U(\mathfrak{g} /[\mathfrak{g}, \mathfrak{g}]) \rightarrow U(\mathfrak{g}) /$ $I_{\text {com }}$. Hence we can prove the kernel of $\varphi$ is equal to $I_{\text {com }}$ by the fact that the composite $\psi \varphi$ is the canonical projection $U(\mathfrak{g}) \rightarrow U(\mathfrak{g}) / I_{\text {com }}$.

Proposition 2.3. Let GK- $\operatorname{dim}_{k} U(\mathfrak{g})$ be the Gelfand-Kirillov dimension of $U(\mathfrak{g})$. Then we have $\mathrm{GK}-\operatorname{dim}_{k} U(\mathfrak{g})=\operatorname{dim}_{k} \mathfrak{g}$.

Proof. See [5, 8.1 .15 (iii)].

Proposition 2.4. Let $\mathfrak{h}$ be an ideal of $\mathfrak{g}$ which is abelian. Let $I_{\mathfrak{h}}$ be the right ideal of $U(\mathfrak{g})$ generated by $\sigma(\mathfrak{h})$, which is a two-sided ideal (cf. [2, 2.2.14]). Then, for any two-sided maximal ideal $\mathfrak{m}$ with $U(\mathfrak{g}) / \mathfrak{m} \cong k$ which contains $I_{\mathfrak{h}}$, we have a Lie algebra isomorphism $\mathfrak{h} \rightarrow I_{\mathfrak{h}} / I_{\mathfrak{h}} \mathfrak{m}$ via $\sigma$. Here the Lie algebra structure of $I_{\mathfrak{h}} / I_{\mathfrak{h}} \mathfrak{m}$ is defined by that of $U(\mathfrak{g})$.

Proof. First, we prove the proposition in the case $\mathfrak{m}=\langle\sigma(\mathfrak{g})\rangle$. Let $g_{1}, \ldots, g_{d}$ be a basis of $\mathfrak{g}$ such that $g_{1}, \ldots, g_{l}$ is a basis of $\mathfrak{h}$. By Poincaré-Birkhoff-Witt theorem, we have

$$
U(\mathfrak{g})=k \oplus \bigoplus_{\substack{s \geq 1 \\ 1 \leq i_{1} \leq \cdots \leq i_{s} \leq d}} k \sigma\left(g_{i_{1}}\right) \cdots \sigma\left(g_{i_{s}}\right) .
$$

Here $\sigma\left(g_{i_{1}}\right) \cdots \sigma\left(g_{i_{s}}\right)\left(1 \leq i_{1} \leq \cdots \leq i_{s} \leq d\right)$ form a $k$-basis of $U$. Since $\mathfrak{h}$ is abelian, we have similar decompositions:

$$
\begin{gathered}
I_{\mathfrak{h}}=\bigoplus_{\substack{s \geq 1 \\
1 \leq i_{1} \leq \cdots \leq i_{s} \leq d \\
i_{1} \leq l}} k \sigma\left(g_{i_{1}}\right) \cdots \sigma\left(g_{i_{s}}\right) ; \\
I_{\mathfrak{h}} \mathfrak{m}=\bigoplus_{\substack{s \geq 2 \\
1 \leq i_{1} \leq \cdots \leq i_{s} \leq d \\
i_{1} \leq l}} k \sigma\left(g_{i_{1}}\right) \cdots \sigma\left(g_{i_{s}}\right) \\
\left.\bigoplus_{1}\right)
\end{gathered}
$$


as $k$-vector spaces. Hence we have an isomorphism

$$
\mathfrak{h} \stackrel{\cong}{\longrightarrow} I_{\mathfrak{h}} / I_{\mathfrak{h}} \mathfrak{m}=\bigoplus_{\substack{s=1 \\ 1 \leq i_{1} \leq l}} k \sigma\left(g_{i_{1}}\right)
$$

via $\sigma$. Using $I_{\mathfrak{h}} \subset \mathfrak{m}$ and $I_{\mathfrak{h}}^{2} \subset I_{\mathfrak{h}} \mathfrak{m}$, one can verify that the Lie algebra structure of $I_{\mathfrak{h}} / I_{\mathfrak{h}} \mathfrak{m}$ is well-defined and abelian.

Next we show the proposition in the general case. Let $\alpha: U(\mathfrak{g}) \rightarrow k$ be a surjective $k$-algebra homomorphism with kernel $\mathfrak{m}$. Using $\alpha$, we have an automorphism $i: U(\mathfrak{g}) \rightarrow U(\mathfrak{g})$ with $i \sigma(g)=\sigma(g)-\alpha(\sigma(g)) \cdot 1$ for all $g \in \mathfrak{g}$. Since $\mathfrak{m}$ contains $I_{\mathfrak{h}}$, the restriction of $i$ to $\sigma(\mathfrak{h})$ is the identity of $\sigma(\mathfrak{h})$. One can easily verify $i(\langle\sigma(\mathfrak{g})\rangle)=\mathfrak{m}$. Hence we have an isomorphism $\mathfrak{h} \rightarrow I_{\mathfrak{h}} / I_{\mathfrak{h}} \mathfrak{m}$ using the isomorphism (1) and a commutative diagram

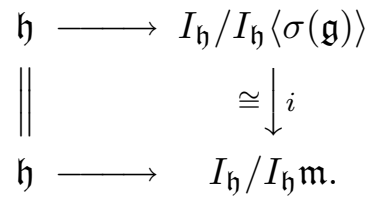

Corollary 2.5. In the notation of Proposition 2.1 , we regard the ideal $I:=$ $I_{[\mathfrak{g}, \mathfrak{g}]}$ as ideal of the underlying Lie algebra $U(\mathfrak{g})$. Assume that $[\mathfrak{g}, \mathfrak{g}]$ is abelian. Then, for any maximal ideal $\mathfrak{m}$ with $U(\mathfrak{g}) / \mathfrak{m} \cong k$, the composite $[\mathfrak{g}, \mathfrak{g}] \stackrel{\sigma}{\longrightarrow} I \stackrel{\mathrm{pr}}{\longrightarrow} I / I \mathfrak{m}$ is an isomorphism of Lie algebras.

Remark 2.6. The composition $[\mathfrak{g}, \mathfrak{g}] \stackrel{\sigma}{\rightarrow} I \stackrel{\mathrm{pr}}{\longrightarrow} I / I \mathfrak{m}$ is surjective for any Lie algebra, but not necessarily injective if $[\mathfrak{g}, \mathfrak{g}]$ is not abelian. For example, consider a simple Lie algebra.

Proposition 2.7. Let $\mathfrak{g}_{0}$ be an ideal of $\mathfrak{g}$. Suppose that there exists a subalgebra $\mathfrak{g}_{1}$ of $\mathfrak{g}$ such that $\mathfrak{g}=\mathfrak{g}_{0} \oplus \mathfrak{g}_{1}$ as k-vector spaces. Then $\mathfrak{g}$ is isomorphic to the semidirect product $\mathfrak{g}_{0} \rtimes \mathfrak{g}_{1}$.

Proof. It is straightforward to show that the $k$-linear map $\mathfrak{g}_{0} \rtimes \mathfrak{g}_{1} \rightarrow \mathfrak{g}$ defined by $\left(g_{0}, g_{1}\right) \mapsto g_{0}+g_{1}$ is a Lie algebra isomorphism.

Proposition 2.8. Let $\mathfrak{g}_{i}$ and $\mathfrak{g}_{i}^{\prime}(i=0,1)$ be Lie algebras and $\mathfrak{g}_{1} \stackrel{d}{\rightarrow} \operatorname{Der}_{k} \mathfrak{g}_{0}$ (resp. $\left.\mathfrak{g}_{1}^{\prime} \stackrel{d^{\prime}}{\longrightarrow} \operatorname{Der}_{k} \mathfrak{g}_{0}^{\prime}\right)$ a derivation of $\mathfrak{g}_{0}$ (resp. $\left.\mathfrak{g}_{0}^{\prime}\right)$. Assume that there exist Lie algebra isomorphisms $\varphi_{0}: \mathfrak{g}_{0} \rightarrow \mathfrak{g}_{0}^{\prime}$ and $\varphi_{1}: \mathfrak{g}_{1} \rightarrow \mathfrak{g}_{1}^{\prime}$ with a commutative diagram

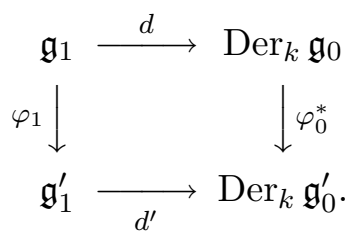


Here $\varphi_{0}^{*}$ is the induced homomorphism by $\varphi_{0}$. Then the semidirect product $\mathfrak{g}_{0} \rtimes \mathfrak{g}_{1}$ is isomorphic to $\mathfrak{g}_{0}^{\prime} \rtimes \mathfrak{g}_{1}^{\prime}$ by $\left(g_{0}, g_{1}\right) \mapsto\left(\varphi_{0}\left(g_{0}\right), \varphi_{1}\left(g_{1}\right)\right)$.

Proof. Straightforward. See [1, Chapitre $1 \S 7]$.

\section{Proof of Theorem 1.1.}

We have only to show that, if $U(\mathfrak{g})$ is isomorphic to $U\left(\mathfrak{g}^{\prime}\right)$, the Lie algebra $\mathfrak{g}$ is isomorphic to $\mathfrak{g}^{\prime}$.

Assume that $U(\mathfrak{g})$ is isomorphic to $U\left(\mathfrak{g}^{\prime}\right)$. We remark that $\operatorname{dim}_{k} \mathfrak{g}=$ $\operatorname{dim}_{k} \mathfrak{g}^{\prime}$ and $\operatorname{dim}_{k}[\mathfrak{g}, \mathfrak{g}]=\operatorname{dim}_{k}\left[\mathfrak{g}^{\prime}, \mathfrak{g}^{\prime}\right]$ by Propositions 2.2 and 2.3 .

In the case of $\operatorname{dim}_{k} \mathfrak{g}=1,2$, the theorem follows from the classification of Lie algebras (e.g., [3, I.4]).

We now assume $\operatorname{dim}_{k} \mathfrak{g}=\operatorname{dim}_{k} \mathfrak{g}^{\prime}=3$. We carry out the proof in each case of $\operatorname{dim}_{k}[\mathfrak{g}, \mathfrak{g}]=0,1,2,3$.

If $\operatorname{dim}_{k}[\mathfrak{g}, \mathfrak{g}]=0$, i.e., $\mathfrak{g}$ is abelian, then the theorem is clear.

Suppose $\operatorname{dim}_{k}[\mathfrak{g}, \mathfrak{g}]=3$. Then one can verify that $\mathfrak{g}$ is simple (cf. [3, I.4]). Hence the theorem follows from a result of Malcolmson [4, Corollary 1].

Finally, we treat the case $\operatorname{dim}_{k}[\mathfrak{g}, \mathfrak{g}]=1,2$. Let $\psi: U(\mathfrak{g}) \rightarrow U\left(\mathfrak{g}^{\prime}\right)$ be an isomorphism. We denote by $\mathfrak{m}$ (resp. $\left.\mathfrak{m}^{\prime}\right)$ the (two-sided) maximal ideal generated by $\sigma(\mathfrak{g})$ (resp. the maximal ideal $\psi(\mathfrak{m})$ ). Let $I:=I_{[\mathfrak{g}, \mathfrak{g}]}$ and $I^{\prime}:=I_{\left[\mathfrak{g}^{\prime}, \mathfrak{g}^{\prime}\right]}$ be as in Proposition 2.1. Note that $[\mathfrak{g}, \mathfrak{g}]$ is abelian in this case (cf. [3, I.4]). By Proposition 2.2 and Corollary 2.5, we have the following commutative diagram:

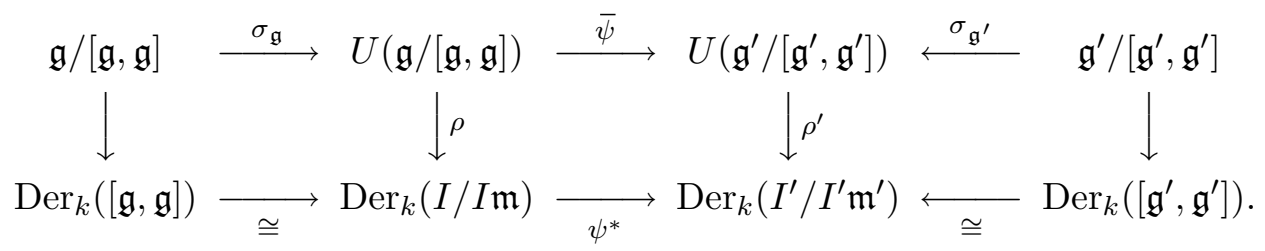

Here $\rho, \rho^{\prime}$ are Lie homomorphisms defined by inner derivation as usual, and $\bar{\psi}\left(\right.$ resp. $\left.\psi^{*}\right)$ is the isomorphism induced by $\psi$.

Suppose $\operatorname{dim}_{k}[\mathfrak{g}, \mathfrak{g}]=1$. Then there are just two isomorphism classes of 3dimensional Lie algebras [3, I.4]: One is nilpotent; the other is not nilpotent. In this case, a Lie algebra $\mathfrak{g}$ is nilpotent if and only if its center contains $[\mathfrak{g}, \mathfrak{g}]$, i.e., the above $\rho$ is trivial for a maximal ideal $\mathfrak{m}$ with $U / \mathfrak{m} \cong k$. The theorem follows from the above diagram.

Next, we suppose $\operatorname{dim}_{k}[\mathfrak{g}, \mathfrak{g}]=2$. Take elements $z \in \mathfrak{g} \backslash[\mathfrak{g}, \mathfrak{g}]$ and $z^{\prime} \in$ $\mathfrak{g}^{\prime} \backslash\left[\mathfrak{g}^{\prime}, \mathfrak{g}^{\prime}\right]$. We denote by $\mathfrak{g}_{1}$ (resp. $\left.\mathfrak{g}_{1}^{\prime}\right)$ the subalgebra of $\mathfrak{g}\left(\right.$ resp. $\left.\mathfrak{g}^{\prime}\right)$ generated by $z$ (resp. $\left.z^{\prime}\right)$. Since

$$
\bar{\psi}\left(\sigma_{\mathfrak{g}}(z \bmod [\mathfrak{g}, \mathfrak{g}])\right)=a \sigma_{\mathfrak{g}^{\prime}}\left(z^{\prime} \bmod \left[\mathfrak{g}^{\prime}, \mathfrak{g}^{\prime}\right]\right)+b \text { for some } a \in k^{*}, b \in k,
$$


we have the following commutative diagram of Lie algebras:

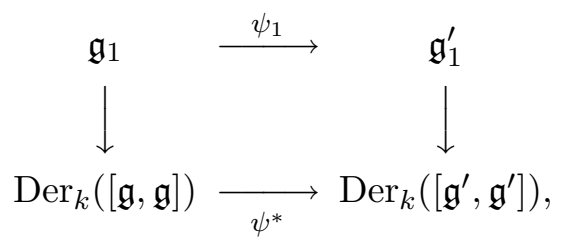

where $\psi_{1}$ maps $z$ to $a z^{\prime}$, and $\psi^{*}$ is the composite of the lower horizontal maps in the above diagram. The theorem follows from Propositions 2.7 and 2.8.

Acknowledgements. The second author is grateful to the third author for introducing this subject and encouraging him. The third author wishes to acknowledge the financial support of Korea Research Foundation made in the program Year 1997.

\section{References}

[1] N. Bourbaki, Éléments de Mathématique, Groupes et Algèbres de Lie, Hermann, Paris, 1971, MR 0271276, Zbl 0213.04103.

[2] J. Dixmier, Enveloping Algebras, North-Holland, Amsterdam, New York, Oxford, 1977, MR 0498740, Zbl 0339.17007.

[3] N. Jacobson, Lie Algebras, Dover, New York, 1962, MR 0143793, Zbl 0121.27504.

[4] P. Malcolmson, Enveloping algebras of simple three-dimensional Lie algebras, J. Algebra, 146 (1992), 210-218, MR 1152441, Zbl 0752.17009.

[5] J.C. McConnell and J.C. Robson, Noncommutative Noetherian Rings, John Wiley and Sons, 1987, MR 0934572, Zbl 0644.16008.

Received November 29, 1999 and revised February 7, 2003.

Department of Mathematics

YEUNGNAM UNIVERSITY

KYONGSAN 712-749

REPUBLIC OF KoREA

E-mail address: jhchun@ynucc.yeungnam.ac.kr

Mathematical Institute

TÔHOKU UNIVERSITY

SENDAI 980-77

JAPAN

E-mail address: kajiwara@math.tohoku.ac.jp

Department of Mathematics

YEUNGNAM UNIVERSITY

KYONGSAN 712-749

REPUBLIC OF KoREA

E-mail address: ljsook@ynucc.yeungnam.ac.kr 